\title{
Effect of a rigid nonpolar solute on the splay, bend elastic constants and on rotational viscosity coefficient of $4,4^{\prime}$ -n-octyl-cyanobiphenyl
}

\author{
Sudeshna DasGupta, Pinku Chattopadhyay and Soumen Kumar Roy
}

November 23, 2018

Department of Physics, Jadavpur University, Calcutta-700 032, INDIA

\begin{abstract}
The effect of a rigid nonpolar non-mesogenic solute, "biphenyl" which is $\mathrm{C}_{6} \mathrm{H}_{5}-\mathrm{C}_{6} \mathrm{H}_{5}$, on the splay and bend elastic constants and on the rotational viscosity coefficient of $4,4^{\prime}$-n-octyl-cyano biphenyl (8CB) is reported. The experiments involve the measurement of voltage dependence of capacitance of a cell filled with the mixture. Anomalous behavior of both $K_{11}$ and $\Delta \epsilon$ near the $N-S_{A}$ transition have been observed.
\end{abstract}

\section{INTRODUCTION}

It is well known that, with few exceptions, the presence of non-mesomorphic solutes in nematics depress the normal nematic-isotropic transition temperature $\left(T_{N I}\right)$ [1]. Moreover, experiments [2, 3] over last few decades have confirmed that the presence of such impurities leads to the formation of a two phase region. This is consistent with the laws of thermodynamics and the first order nature of the $N-I$ transition. Two types of models have proved to be generally useful for investigating $N-I$ transitions in dilute solutions. The first is a lattice model [4. 5] which takes into account the role of intermolecular repulsion or the steric effect. The other [6] is the mean field theory of the Maier-Saupe type which takes into account the anisotropic intermolecular attraction or dispersion forces. More recently Mukherjee [7] has explained the behavior of the two phase region using the phenomenological Landau de Gennes theory.

The role of non mesogenic impurities on a mesogen which exhibits a smectic phase as well, has not received a great deal of attention of either experimentalists or theorists. Here too, in favourable circumstances, remains a possibility of the existence of a two phase region near the smectic transition $[8]$. 
In this paper we report the results of measurements carried out on a mixture of biphenyl and 4, 4'-n-octyl-cyano biphenyl (8CB). The latter which has a strong polar group and a long alkyl chain has received considerable attention of experimentalists over last few decades [9, 10, 11, 12] and is known to exhibit a first order $S_{A}-N$ transition. The biphenyl $+8 \mathrm{CB}$ system to our knowledge, has not been studied before and is a natural and interesting choice in its own merit since the solute, which is chemically $\mathrm{C}_{6} \mathrm{H}_{5}-\mathrm{C}_{6} \mathrm{H}_{5}$, is a non-mesogenic, non-polar, rigid molecule and is nothing but $8 \mathrm{CB}$ deprived of its cyano group and the flexible alkyl chain. We have observed that biphenyl is easily miscible with $8 \mathrm{CB}$ and lowers both $T_{A N}$ and $T_{N I}$ by an amount which depends on the impurity concentration. The range of the nematic phase i.e., $\left(T_{N I}-T_{A N}\right)$ however is not significantly altered. The effect of this rod-like non-polar impurity on the dielectric anisotropy, the Frank elastic constants (splay and bend) and on the effective rotational viscosity coefficient of pure $8 \mathrm{CB}$ has been reported here. To our knowledge, no measurement has yet been reported on the effect of impurities on the elastic constants and the viscosity coefficient of a nematic. The experiments we have carried out involve electric field induced Freedericksz transition and consist of two parts. The dielectric anisotropy and the Frank elastic constants are extracted from a set of measurements of the voltage dependence of capacitance of a sample cell filled with the $8 \mathrm{CB}$ and biphenyl mixture. For the determination of the rotational viscosity coefficient a method based on dynamic Freedericksz transition is used. Interesting features have emerged from these measurements and the splay elastic constant is seen to exhibit anomalous behavior in the vicinity of the $S_{A}-N$ transitions for the impurity concentration exceeding a certain value.

\section{PRINCIPLE OF THE METHOD}

The splay elastic constant $K_{11}$ is related to the Freedericksz threshold voltage $V_{t h}$ by the relation

$$
K_{11}=\epsilon_{0} \Delta \epsilon / \pi^{2} V_{t h}^{2}
$$

where $\epsilon_{0}$ is the permittivity of free space and $\Delta \epsilon=\epsilon_{\|}-\epsilon_{\perp}$, the dielectric anisotropy of the sample. The method of determination of $\Delta \epsilon$ is described below.

The exact relationship between the cell capacitance $C$ and the voltage $V$ applied across the cell was obtained by Gruler [13]

$$
\frac{V}{V_{t h}}=\frac{2}{\pi}\left(1+\gamma \sin ^{2} \phi_{m}\right)^{1 / 2} \int_{0}^{\phi_{m}}\left[\frac{\left(1+\kappa \sin ^{2} \phi\right)}{\left(1+\gamma \sin ^{2} \phi\right)\left(\sin ^{2} \phi_{m}-\sin ^{2} \phi\right)}\right]^{1 / 2} d \phi
$$

and 


$$
\frac{C}{C_{\perp}}=\frac{\int_{0}^{\phi_{m}}\left[\frac{\left(1+\kappa \sin ^{2} \phi\right)\left(1+\gamma \sin ^{2} \phi\right)}{\left(\sin ^{2} \phi_{m}-\sin ^{2} \phi\right)}\right]^{1 / 2} d \phi}{\int_{0}^{\phi_{m}}\left[\frac{\left(1+\kappa \sin ^{2} \phi\right)}{\left(1+\gamma \sin ^{2} \phi\right)\left(\sin ^{2} \phi_{m}-\sin ^{2} \phi\right)}\right]^{1 / 2} d \phi}
$$

where $\kappa=K_{33} / K_{11}-1$ ( $K_{33}$ being the bend elastic constant), $\gamma=\epsilon_{\|} / \epsilon_{\perp}-1, \phi$ is the tilt angle made by the director with a direction parallel to the cell walls, $\phi_{m}$ is the tilt angle at the center of the cell and $C_{\perp}$ is the capacitance of the cell when the liquid crystal molecules are homogeneously aligned, that is, before the onset of Freedericksz transition when the voltage applied is lower than the threshold voltage. These equations can be combined to yield

$\frac{C-C_{\perp}}{C_{\perp}}=\gamma-\frac{2 \gamma}{\pi}\left(1+\gamma \sin ^{2} \phi_{m}\right)^{1 / 2} \frac{V_{t h}}{V} \int_{0}^{\sin \phi_{m}}\left[\frac{\left(1+\kappa x^{2}\right)\left(1-x^{2}\right)}{\left(1+\gamma x^{2}\right)\left(\sin ^{2} \phi_{m}-x^{2}\right)}\right]^{1 / 2} d x$

When the applied voltage is much higher that the threshold voltage, the director at the center of the cell becomes perpendicular to the cell walls and $\phi_{m}=\pi / 2$. Then the above equation reduces to

$$
\frac{C-C_{\perp}}{C_{\perp}}=\gamma-\frac{2 \gamma}{\pi}(1+\gamma)^{1 / 2} \frac{V_{t h}}{V} \int_{0}^{1}\left[\frac{\left(1+\kappa x^{2}\right)}{\left(1+\gamma x^{2}\right)}\right]^{1 / 2} d x
$$

or dividing by $\gamma$

$$
\frac{C-C_{\perp}}{C_{\|}-C_{\perp}}=C_{R}=1-\frac{2}{\pi}(1+\gamma)^{1 / 2} \frac{V_{t h}}{V} \int_{0}^{1}\left[\frac{\left(1+\kappa x^{2}\right)}{\left(1+\gamma x^{2}\right)}\right]^{1 / 2} d x
$$

where $C_{R}$ may be called the reduced capacitance. $C_{\|}$is the capacitance of the cell when the nematic is homeotropically oriented, i.e., the value of $C$ in the limit $1 / V \rightarrow 0$. $\epsilon_{\|}$and $\epsilon_{\perp}$ may be obtained by dividing $C_{\|}$and $C_{\perp}$ by the empty cell capacitance $C_{0}$ respectively. In the above equation use has been made of the relation $\gamma=\left(C_{\|}-C_{\perp}\right) / C_{\perp}$.

Thus equation (5) predicts that a plot of $\left(C-C_{\perp}\right) / C_{\perp}$ against $1 / V$ for $V \gg V_{t h}$ should be linear and the extrapolated value of the ordinate for $1 / V$ $\rightarrow 0$ should directly provide the value of $\gamma=\Delta \epsilon / \epsilon_{\perp}$. This procedure for obtaining $\gamma$ was first suggested by Meyerhofer 14 .

The variation of capacitance with applied voltage was fitted against equations (2) and (3) to obtain $V_{t h}$ and $\kappa$. We then used (11) to calculate $K_{11}$ and hence $K_{33}$. This method for obtaining $V_{t h}$ and $\kappa$ was suggested by Morris et al [11].

The viscous behavior of a nematic can be obtained by studying the response to a sudden change of an applied magnetic or electric field which is normally stronger than the Freedericksz-threshold value. In case of deformations involving a pure twist there is no hydrodynamic flow. The molecules merely rotate without any translational motion and the analysis is rather simple $[15]$. The situation is far more complicated in case of a splay geometry which we have studied since 
the director reorientation is now accompanied by a hydrodynamic flow. The gradient of the angular velocity of the director produces a backflow motion, first demonstrated by Pieranski [16] giving rise to a frictional torque. The sudden removal of an external field in this case would result in a director relaxation time

$$
\tau=\gamma_{1}^{*} d^{2} /\left(\pi^{2} K_{11}\right)
$$

where $\gamma_{1}^{*}$ is the effective rotational viscosity coefficient, $d$ is the cell thickness and $K_{11}$ is the splay elastic constant. In practice $\gamma_{1}^{*}$ is about $10 \%$ less than the rotational viscosity coefficient $\gamma_{1}$.

We have determined the relaxation time $\tau$ by measuring the decay of capacitance in a nematic cell. The details are given in the next section.

\section{DETAILS OF THE EXPERIMENTAL METHOD}

We have used a Hewlett-Packard LCR meter HP 4274A to measure the capacitance of the sample cell. The instrument employs the so called "auto balancing bridge method" to measure both the real and complex parts of impedance simultaneously. The voltage was varied from $0.1 \mathrm{~V}$ to $5 \mathrm{~V}$ at a frequency of $1 \mathrm{KHz}$. The sample cells, which were of very high precision, were obtained from Displaytech, USA. The cells consisting of two ITO coated glass plates with a spacing of $4 \mu \mathrm{m}$, and an active area of $0.26 \mathrm{~cm}^{2}$, had brushed polyimide treatment to ensure planar orientation. They also had a guard ring incorporated in them to minimize fringe electric fields. The temperature of the cells were controlled to within $\pm 0.1 K$ by placing the cells within a Mettler hot stage FP 82 . The texture of the samples were viewed through a Leitz polarizing microscope and photographs were taken with the help of Photoautomat. Both $8 \mathrm{CB}$ and biphenyl were obtained from Merck and were used without further purification.

The samples which we have used were mixtures of various concentrations of biphenyl, ranging from $0.4 \%$ to $6.4 \%$, in pure $8 \mathrm{CB}$. The smectic to nematic transition temperature, $T_{A N}$ and the nematic to isotropic transition temperature $T_{N I}$ were determined from texture studies of the samples. Various photographs were taken at different stages of transition. In the capacitance measurement experiments the probe voltage of the LCR meter HP 4274A provided the aligning a.c. electric field. The voltage was varied from $0.1 \mathrm{~V}$ to $5 \mathrm{~V}$ at a frequency of $1 \mathrm{KHz}$ in the $C_{P}-G$ mode of the instrument. In the neighborhood of Freedericksz transition readings were taken at intervals of $10 \mathrm{mV}$. The samples were filled in the cells and the $\mathrm{C}-\mathrm{V}$ variation was recorded at a large number of temperatures ranging from the smectic to nematic transition temperature, $T_{A N}$, to a few degrees beyond the nematic to isotropic transition temperature, $T_{N I}$. It must be noted that all measurements were carried out as the temperatures of the sample were increased gradually starting from $T<T_{A N}$ and none of the measurements were taken during cooling. Fig 1 i shows a few capacitance-voltage variations of the mixture with biphenyl concentration $4.59 \%$ at temperatures near the two transitions $S_{A}-N$ and $N-I$ and at an intermediate temperature. There is 
always a need to have a refinement in the value of $V_{t h}$ as the C-V curves obtained in a polyimide cell usually do not exhibit a very sharp threshold of Freedericksz transition so as to yield a precise value of $V_{t h}$ from the data directly. From the $\mathrm{C}-\mathrm{V}$ data $\epsilon_{\perp}$ and $\epsilon_{\|}$were calculated and subsequently $V_{t h}$ and $\kappa$ were determined as described below. $\epsilon_{\perp}$ was determined directly from the value of capacitance at voltages lower than the threshold voltage, (before the onset of the Freedericksz transition) by dividing the capacitance, $C_{\perp}$ by the empty cell capacitance $C_{0} . \epsilon_{\|}$ was obtained by plotting $C$ against $1 / V$ for the higher voltage range $(4.0-5.0 \mathrm{~V})$. The extrapolated value of capacitance in the limit as $1 / V \rightarrow 0$ gives $C_{\|}$which when divided by $C_{0}$ gives $\epsilon_{\|}$.

A direct measurement of $C_{\|}$was also carried out to check the reliability of the value of $\gamma$ obtained by the above method. A homeotropic alignment was obtained in the ITO coated glass cells using CTAB (cetyl trimethyl ammonium bromide) and the capacitance of the cell was measured while a $10 \mathrm{KG}$ magnetic field was applied across the cell. The values of $\gamma$ thus obtained never differed from the $\gamma$ 's obtained from the $1 / V$-extrapolation method by more than $0.1 \%$.

A two parameter non-linear least square fit for finding $V_{t h}$ and $\kappa$ worked in the following manner. Starting values of $V_{t h}$ and $\gamma$ were taken directly from the experiment. (2) was then used to obtain the $\phi_{m}$ 's for all values of $\mathrm{V}$ for which measurements of capacitance were carried out. The $\phi_{m}$ 's thus obtained were used in (3) to obtain $\mathrm{C}$. The error which was minimized in the least square programme is $\sum_{i=1}^{n}\left(C_{i}^{\text {expt }}-C_{i}\right)^{2}$ where $C_{i}^{\text {expt }}$ is the measured value of the capacitance at $\mathrm{V}=V_{i}$ and $C_{i}$ is the value of capacitance obtained by solving (3), $\mathrm{n}$ being the number of data points for a particular temperature. The final values of $V_{t h}$ never differed a great deal from the input values.

For the rotational viscosity coefficient measurements, we have used a HewlettPackard impedance gain-phase analyser HP 4194A in the programmable mode to record the decay of capacitance with time. An integration time of $500 \mu \mathrm{sec}$ was chosen which resulted in an interval of 5 msec between the successive readings at the operating frequency which was fixed at $10 \mathrm{KHz}$. With our instrument a delay time, in multiples of $1 \mathrm{msec}$, could be introduced between the readings but in this case we did not introduce any delay since the decay of capacitance was found to be pretty fast. The a.c. probe voltage across the sample cell was held fixed at $0.3 \mathrm{~V}$. A low frequency a.c. voltage about 2 volts peak to peak was applied across the cell for about 30 secs. It was switched off and the transient (decaying) capacitance of the cell, was captured as a function of time by the HP 4194A. A plot of this decay of capacitance with time which was always found to be exponential was then dumped into a HP $7475 \mathrm{~A}$ plotter which was connected to the impedance analyser over a HPIB bus. From the plots thus obtained for different concentration of biphenyl mixtures, at different temperatures, the time constant, $\tau$ was calculated and using the values of $K_{11}$ obtained from the static capacitance measurements, the effective rotational viscosity coefficient, $\gamma_{1}^{*}$ was evaluated. 


\section{RESULTS AND DISCUSSIONS}

The transition temperatures $T_{N I}$ (upper), $T_{N I}$ (lower) and $T_{A N}$ were determined from optical texture studies and the results are presented in Table 11 and Fig.2. The $T_{N I}$ (upper) is the temperature at which the field of view of the polarizing microscope (with crossed polarizer and analyzer) becomes completely dark. All real nematics contain impurities and Rosenblatt [17] has observed in $8 \mathrm{CB}$ a two phase $(N+I)$ region of temperature of width $13 m K$ in absence of an electric field. Needless to say, with our temperature resolution of $0.1 \mathrm{~K}$, we were unable to confirm this. The width of the two phase region was seen to increase with the concentration of biphenyl and for the maximum concentration of $6.4 \%$ this was $1.6 \mathrm{~K}$. We were unable to work with higher impurity concentration because of non-availability of an experimental arrangement for producing sub-ambient temperatures. All the transition temperatures reported in Table 1 were determined without the presence of any external field. However, we confirmed that with the highest electric field we applied across the sample cells, namely $1.25 \times 10^{4} \mathrm{volt} / \mathrm{cm}$, there was no noticeable change in any of the transition temperatures.

The temperature dependence of the dielectric anisotropy for the concentration (c) of biphenyl ranging from 0 to $4.59 \%$ is shown in Fig.3. In pure $8 \mathrm{CB}$ and for all mixtures we have studied $\Delta \epsilon$, in the neighborhood of $T_{N I}$, shows the same feature in that it disappears at $T_{N I}$ (upper). At this particular temperature the field of view in the microscope with crossed polars is completely dark and hence the phase is isotropic. Values of the dielectric constant $\epsilon_{i s o}$ obtained well within the isotropic phase show a gradual increase with temperature for pure $8 \mathrm{CB}$ as well as for all mixtures we have studied.

Focussing on the behavior of $\Delta \epsilon$ as $T_{A N}$ is approached from the higher temperature side we find that for biphenyl concentration, c upto $2.00 \% \Delta \epsilon$ in mixtures behaves the same way as that in pure $8 \mathrm{CB}$, namely that it shows a sharp increase. However, for impurity concentrations of $3.30 \%$ and higher, $\Delta \epsilon$ goes down sharply, while always remaining positive (at least in the temperature range we have explored). We have noted that the decrease in $\Delta \epsilon$ results from a reduction of $\epsilon_{\|},\left(\epsilon_{\perp}\right.$ remaining fairly constant $)$ as $T_{A N}$ is approached. A similar decrease in $\epsilon_{\|}$as $T_{A N}$ is approached has been observed in pure $p, p^{\prime}$ diheptylazoxybenzene where the $N-S_{A}$ transition is almost second order. This has been attributed to the presence of pretransitional effects within the nematic phase 18.

The temperature and concentration dependence of the splay elastic constant $K_{11}$ is shown in Fig. 4 . The ratio $\kappa$ was seen to approach the value 0 and stay around that value at and around transition but then it was seen to increase again with increase in temperature. The plot of bend elastic constant $K_{33}$ with

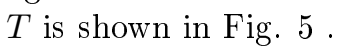

The bend elastic constant $K_{33}$ is seen to diverge as the smectic phase is approached from the nematic side. The behavior was of the type $\Delta K_{33} \propto$ $t^{\nu}$, where $\Delta K_{33}$ is the difference [11] between $K_{33}$ and its nematic part, $t=$ $\left(T / T_{A N}^{*}-1\right)$ and $\nu$ is the critical exponent. We found that for all the samples 
studied $\nu$ is $1.0 \pm 0.1$ which is in agreement with the exponent obtained by Morris et al [11] in pure $8 \mathrm{CB}$. It must be recalled, however, that anisotropic scaling laws, predict that $\Delta K_{33}$ should vary as the correlation length $\xi_{\|}$both above and below $T_{A N}$ and this should result in an exponent $\nu_{\|}$, which from $\mathrm{X}$-ray scattering experiments in many samples turn out to be $\simeq 0.57-0.75$ [8]. But the temperature $T_{A N}^{*}$ at which $K_{33}$ diverges is slightly higher by $\sim 1^{0} K$ than $T_{A N}$ for the mixtures and for ease of eye, indicated by the vertical lines in Fig. . . We are however unable to say if the weakly first order $S_{A}-N$ transition in pure $8 \mathrm{CB}$ approaches a second order transition in the mixtures and this needs more detail studies.

On plotting $K_{11}$ against $\gamma$ we find that the variation for all the concentrations lie nearly on a universal curve, as shown in Fig.6. The deviations which are seen in the figure are mainly the points for the higher concentration mixtures which lie in the vicinity of the two transitions.

The abrupt decrease in $\epsilon_{\|}$as the smectic phase is approached, seems to occur at relatively higher concentrations of biphenyl and is totally absent in pure $8 \mathrm{CB}$ and in the mixtures of low impurity concentration. The optical texture studies we have performed fail to reveal the existence of any smectic phase other than smectic A in the mixtures at low temperatures. It may however be conjectured that the increase in the proportion of biphenyl in the mixture, though small, is leading to a type of ordering, cybotactic or otherwise, of the $8 \mathrm{CB}$ molecules where the contribution of the permanent dipole moment of the $-C N$ group to $\epsilon_{\|}$is greatly reduced.

The decrease in $\epsilon_{\|}$which suggests the existence of pre-transitional smectic effects within the nematic phase occurs only for biphenyl concentrations greater than a limiting value. It may be noted that the ratio $T_{A N} / T_{N I}$ changes from 0.9779 in pure $8 \mathrm{CB}$ to 0.9735 in the $4.59 \%$ biphenyl- $8 \mathrm{CB}$ mixture. It may be pointed out that the decrease in this ratio, though marginal, is believed to favor a second order $S_{A}-N$ transition.

The temperature dependence of the rotational viscosity coefficient for the concentration of biphenyl ranging from 0 to $4.59 \%$ is shown in Fig.7. The $T_{N I}$ (upper) is the temperature at which the field of view of the polarizing microscope (with crossed polarizer and analyser) becomes completely dark. In pure 8CB and for all the mixtures $\gamma_{1}^{*}$ in the neighborhood of $T_{N I}$, shows the same feature in that it disappears at or before $T_{N I}$ (upper). However, as we approach $T_{A N}$ from the higher temperature side we find that for impurity concentrations upto $2.00 \% \gamma_{1}^{*}$ shows a sharp increase as in pure $8 \mathrm{CB}$ while for impurity concentration of and higher than $3.30 \%, \gamma_{1}^{*}$ is seen to decrease sharply. We are not aware of any systematic study involving te effect of non-mesogenic solutes on the rotational viscosity coefficient of the nematics in the neighborhood of nematicsmectic transition. The Miesowicz viscosities of a mixture of $8 \mathrm{OCB}$ and $4 \mathrm{TPB}$ (exhibiting the nematic-smectic transition) were recently investigated by Janik et al [19] using a Miesowicz viscometer.

Fig. 8 shows the plot of $1 / T$ vs. $\ln \left(\gamma_{1}^{*} / \Delta \epsilon\right)$. The dielectric anisotropy $\Delta \epsilon$ is roughly proportional to the long range nematic order parameter. For pure as well as all mixtures the plot of $1 / T$ vs. $\ln \left(\gamma_{1}^{*} / \Delta \epsilon\right)$ gives a straight line. The same 


\begin{tabular}{|c|c|c|c|}
\hline Concentration (\%) & $T_{A N}$ & $T_{N I}$ (lower) & $T_{N I}$ (upper) \\
\hline \hline 0.00 & 33.2 & 40.1 & 40.2 \\
\hline 0.40 & 32.8 & 39.8 & 39.9 \\
\hline 0.90 & 31.0 & 38.1 & 38.4 \\
\hline 1.58 & 29.9 & 37.2 & 37.6 \\
\hline 2.00 & 29.6 & 36.5 & 37.1 \\
\hline 3.30 & 27.1 & 34.3 & 35.0 \\
\hline 4.00 & 25.5 & 33.1 & 33.9 \\
\hline 4.59 & 24.9 & 32.2 & 33.2 \\
\hline 6.40 & - & 28.2 & 29.8 \\
\hline
\end{tabular}

Table 1: Variation of phase transition temperatures $T_{A N}$ and $T_{N I}$ with concentration of biphenyl in $8 \mathrm{CB}$

sort of dependence has been obtained by Gasparoux and Prost [20], Heppke and Schneider [21] for MBBA and by Prost et al [22], for a mixture of two isomers of $p-$ methoxy $-p^{\prime}-$ butylazoxybenzene. Close to $T_{N I}$ and $T_{A N}$ there are deviations and those points have not been shown in the figure. From the slope of these plots the activation energy for diffusion [15] was calculated and has been shown in Table 2.

\section{CONCLUSIONS}

To summarize it can be stated that the presence of the rigid, non-polar and nonmesogenic solute biphenyl in $8 \mathrm{CB}$ results in a change in the dielectric anisotropy $\Delta \epsilon$, the splay elastic constant $K_{11}$ and the effective rotational viscosity coefficient $\gamma_{1}^{*}$. In the neighborhood of the nematic-smectic A transition for the concentration of biphenyl $\geq 3.3 \%$ the changes in these quantities have been found to be anomalous in that, instead of the usual rapid increase observed on cooling, $\Delta \epsilon, K_{11}$ and $\gamma_{1}^{*}$ sharply decrease. This may perhaps be attributed to the presence of pretransitional effects within the nematic phase. From optical texture studies we could not identify any new smectic phase in the mixtures other than what is found in $8 \mathrm{CB}$. The bend elastic constant $K_{33}$ in all the mixtures was found to diverge as the smectic phase was approached and the critical exponent was found to be the same as that observed for pure $8 \mathrm{CB}$, although the temperatures at which the divergences occur were slightly higher than the respective values of $T_{A N}$. More elaborate studies involving DSC, X-ray diffraction and refractive index measurements (to obtain the long range nematic order parameter) may perhaps reveal more about what is actually happening in the mixtures near the smectic A-nematic transition particularly for those containing relatively higher biphenyl concentration. 


\begin{tabular}{|c|c|}
\hline Concentration (\%) & Activation Energy (eV) \\
\hline \hline 0.00 & 0.74 \\
\hline 0.40 & 0.62 \\
\hline 0.90 & 0.73 \\
\hline 1.58 & 0.54 \\
\hline 2.00 & 0.53 \\
\hline 3.30 & 0.32 \\
\hline 4.00 & 0.40 \\
\hline 4.59 & 0.29 \\
\hline
\end{tabular}

Table 2: Variation of activation energies with concentration of biphenyl in 8CB

\section{ACKNOWLEDGEMENTS}

The authors acknowledge valuable discussions with Dr. A. DasGupta, Dr. P.K. Mukherjee and Dr. K. Mukhopadhyay. The research was supported by DST (grant no. SP/S2/M-20/95). SDG acknowledges the award of a fellowship.

\section{References}

[1] D. E. Martire et al. J. Chem. Phys., 64:1456, 1976.

[2] H. Peterson and D. E. Martire. Mol. Cryst. Liq. Cryst., 25:89, 1974.

[3] B. Kroberg, D. Gilson, and D. Patterson. J. Chem. Soc., Faraday II, $72: 1673,1976$.

[4] M. Cotter and D. E. Martire. Mol. Cryst. Liq. Cryst., 7:295, 1969.

[5] M. Cotter. Mol. Cryst. Liq. Cryst., 35:33, 1976.

[6] W. Maier and A. Saupe. Z. Naturforsch, 14a:882, 1959.

[7] P. K. Mukherjee. Liq. Cryst., 22:239, 1997.

[8] P. G. de Gennes and J. Prost. The Physics of Liquid Crystals. Oxford Science Publications, 1993.

[9] P. P. Karat and N. V. Madhusudana. Mol. Cryst. Liq. Cryst., 40:239, 1977.

[10] M. Bradshaw, E. Raynes, J. Bunning, and T. E. Faber. J. Physique, 46:1513, 1985.

[11] S. W. Morris, P. Palffy-Muhoray, and D. Balzarini. Mol. Cryst. Liq. Cryst., 139:263, 1986.

[12] P. Chattopadhyay and S. K. Roy. Mol. Cryst. Liq. Cryst., 89:257, 1994.

[13] H. Gruler, T. J. Scheffer, and G. Meier. Z. Naturforsch, 72a:966, 1972. 


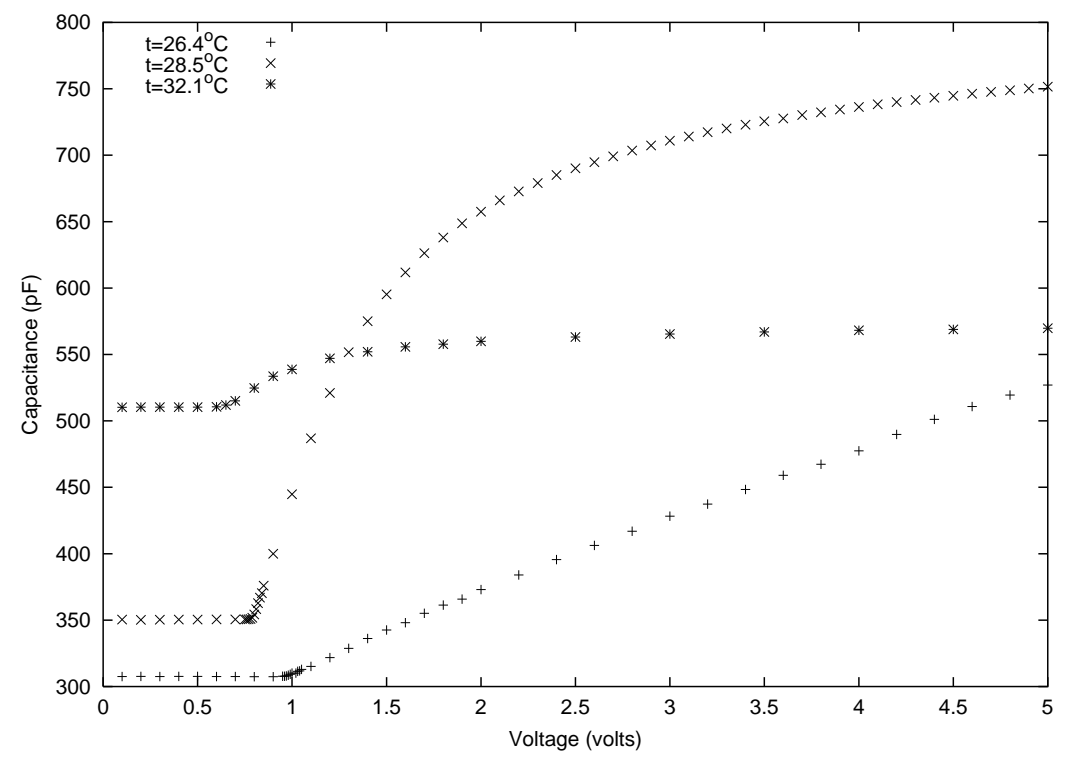

Figure 1: Variation of capacitance $C$ with voltage $V$ for different temperatures $t$ for a $4.59 \%$ mixture of biphenyl in $8 \mathrm{CB}$.

[14] D. Meyerhofer. J. Appl. Phys., 46:5084, 1975.

[15] W. H. de Jeu. Physical Properties of Liquid Crystalline Materials, volume 1. Gordon and Breach Science Publishers.

[16] P. Pieranski, F. Brochard, and E. Guyon. J.Phys, 34:35, 1973.

[17] C. Rosenblatt. Phys. Rev. A, 25:1239, 1982.

[18] W. H. de Jeu, W. J. A. Goosens, and P. Bordewijk. J. Chem. Phys., 61:1985, 1974.

[19] J. Janik, J. K. Moscicki, K. Czuprynski, and R. Dabrowski. Phys. Rev. E, 58:3251, 1998.

[20] H. Gasparoux and J. Prost. J. Phys. Paris, 32:953, 1971.

[21] G. Heppke and F. Schneider. Z. Naturforsch, 27:976, 1972.

[22] J. Prost, G. Sigand, and B. Regaya. J. Phys. Lett., Paris, 37:L341, 1976. 


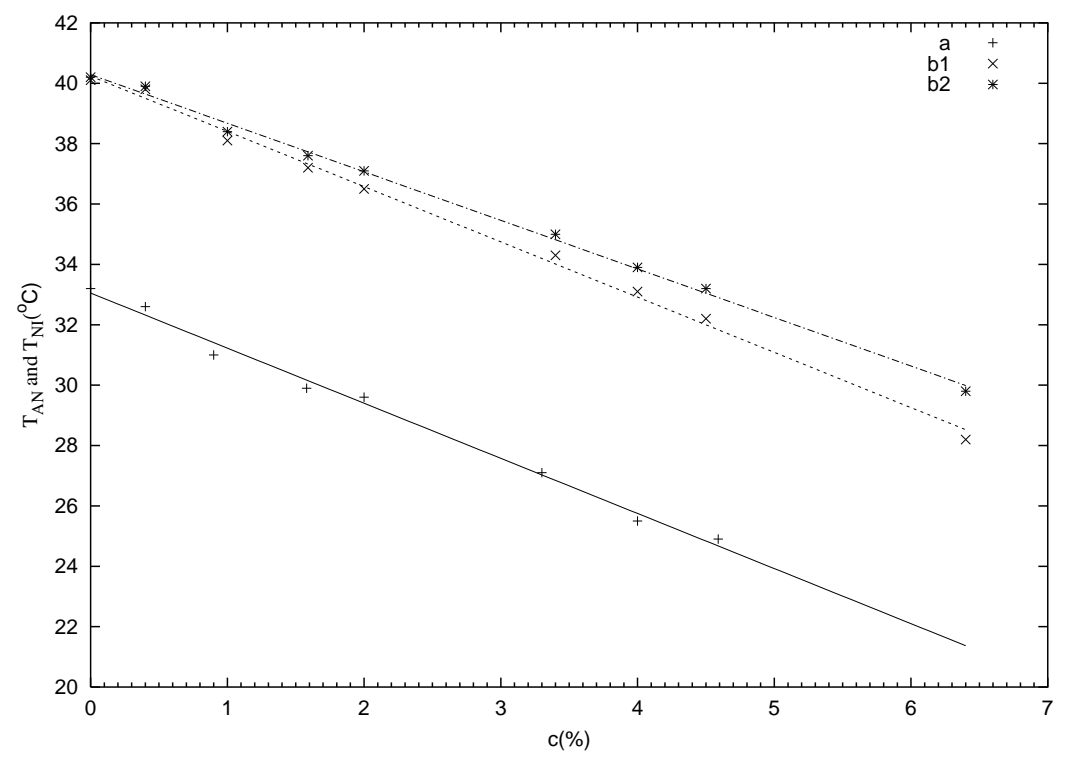

Figure 2: Variation of $T_{N I}$ and $T_{A N}$ with concentration of biphenyl (c) . (a $=T_{A N}, \mathrm{~b} 1=T_{N I}$ (lower), b2 $=T_{N I}$ (upper))

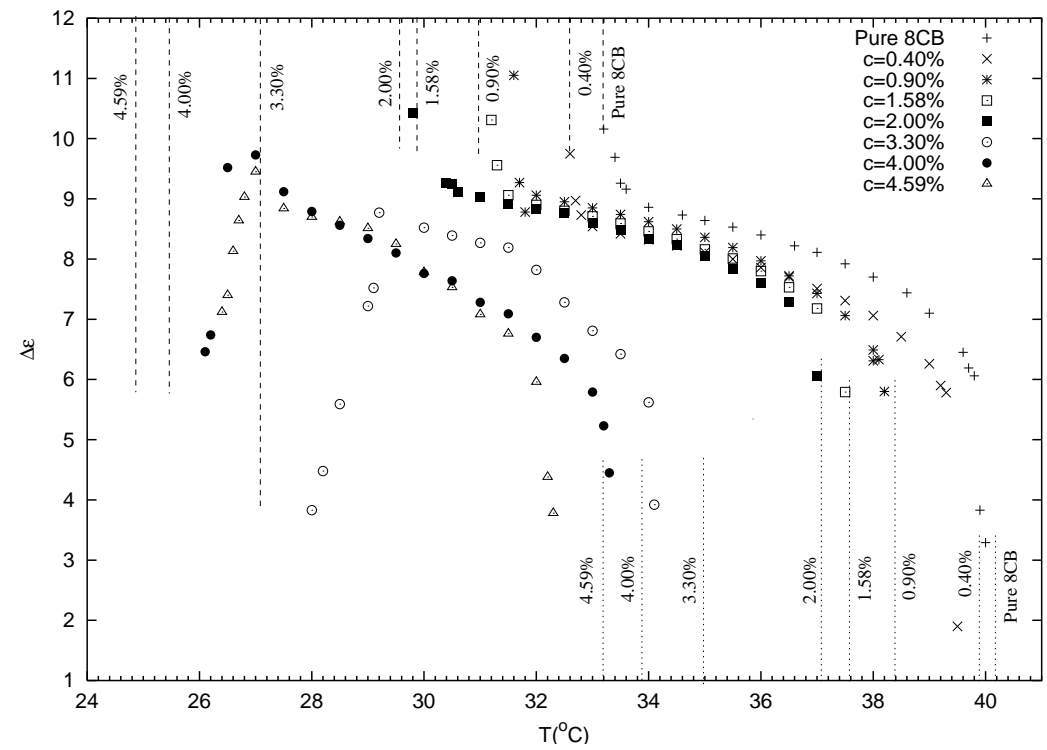

Figure 3: Variation of $\Delta \epsilon$ with $T$ for different concentrations of biphenyl in $8 \mathrm{CB}$. The vertical lines denote the transition temperatures $T_{A N}$ and $T_{N I}$ for different concentrations of biphenyl in $8 \mathrm{CB}$ as shown in Table 1 . 


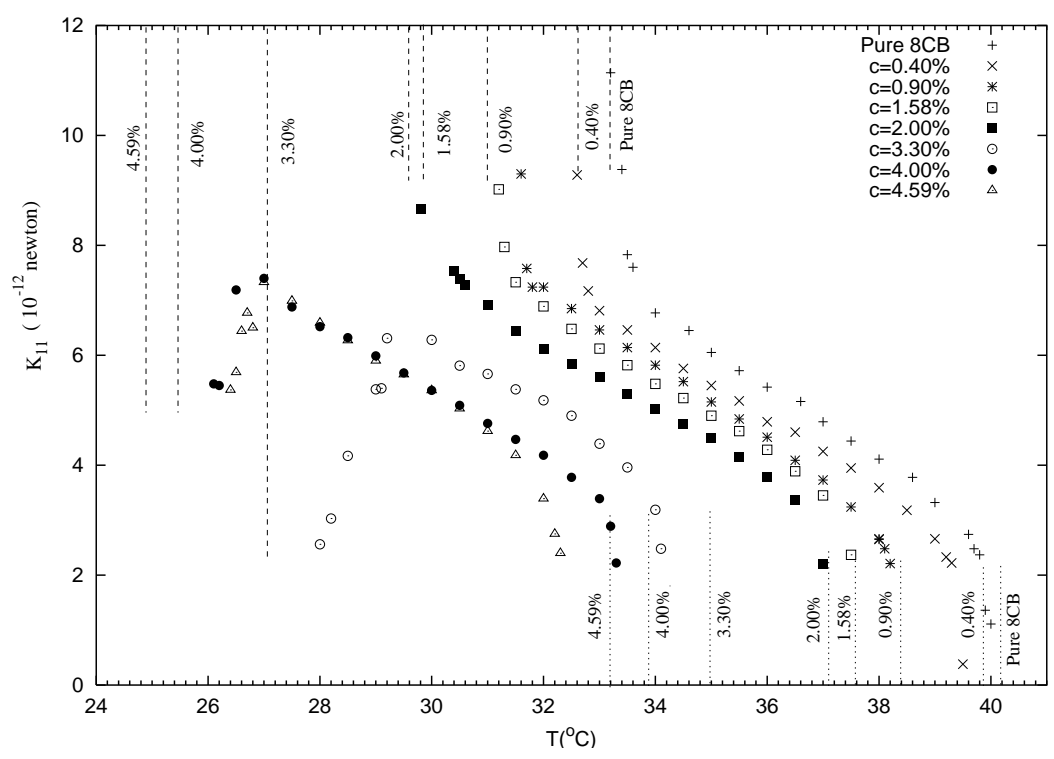

Figure 4: Variation of $K_{11}$ with $T$ for different concentrations of biphenyl in $8 \mathrm{CB}$. The vertical lines denote the transition temperatures $T_{A N}$ and $T_{N I}$ for different concentrations of biphenyl in $8 \mathrm{CB}$ as shown in Table 1 .

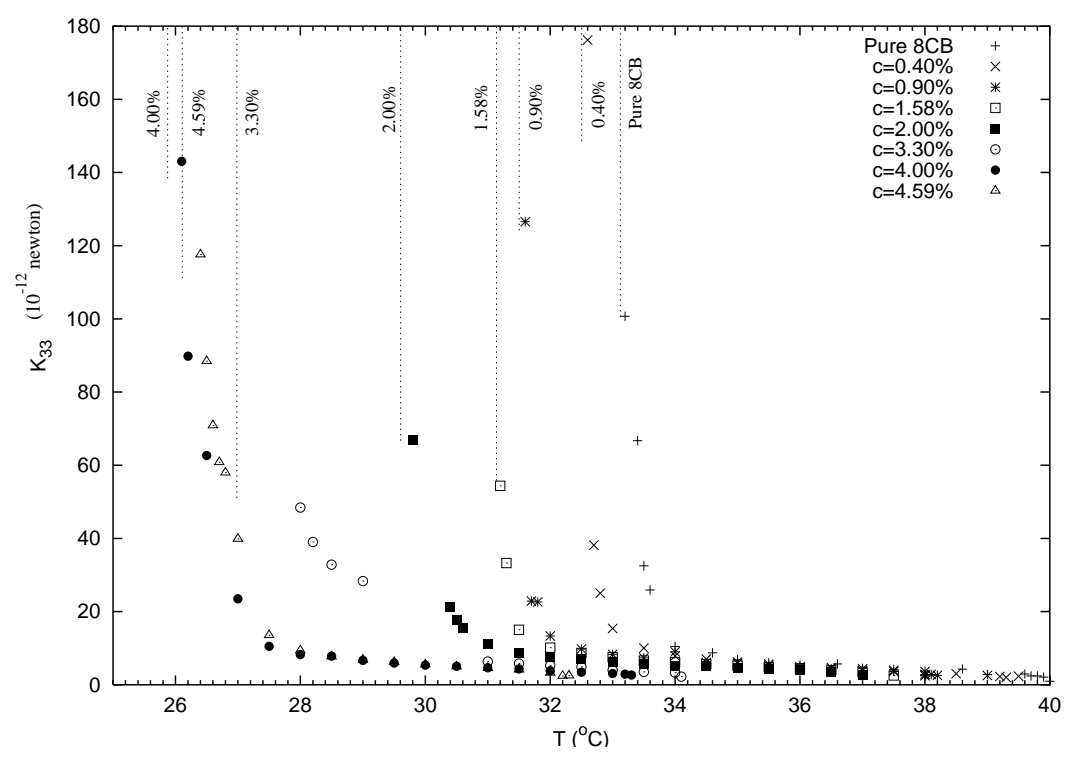

Figure 5: Variation of $K_{33}$ with $T$ for different concentrations of biphenyl in $8 \mathrm{CB}$ 


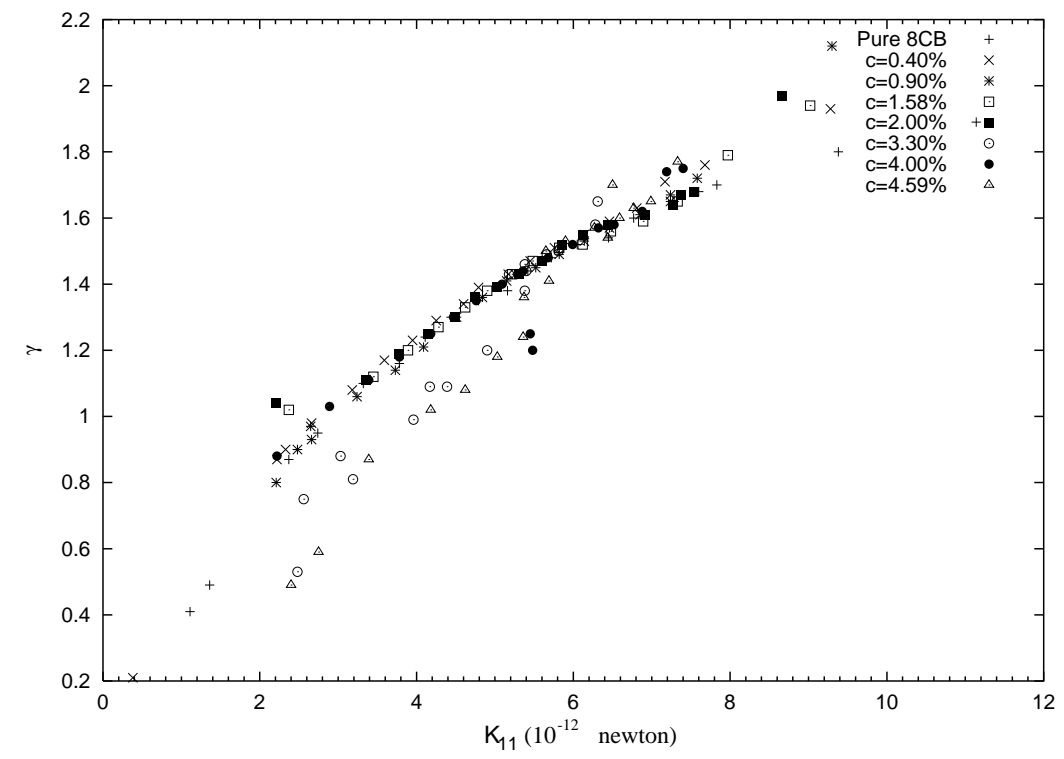

Figure 6: Variation of $K_{11}$ with $\gamma$ for different concentrations of biphenyl in $8 \mathrm{CB}$

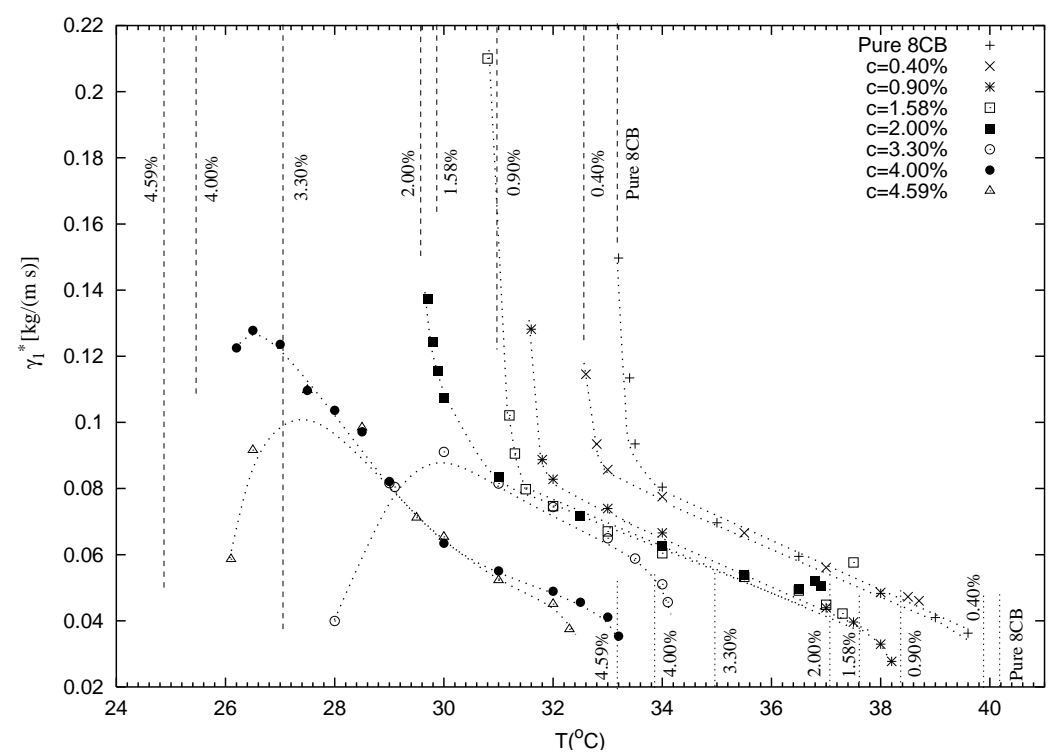

Figure 7: Variation of $\gamma_{1}^{*}$ with $T$ for different concentrations of biphenyl in 8CB. The vertical lines denote the transition temperatures $T_{A N}$ and $T_{N I}$ 


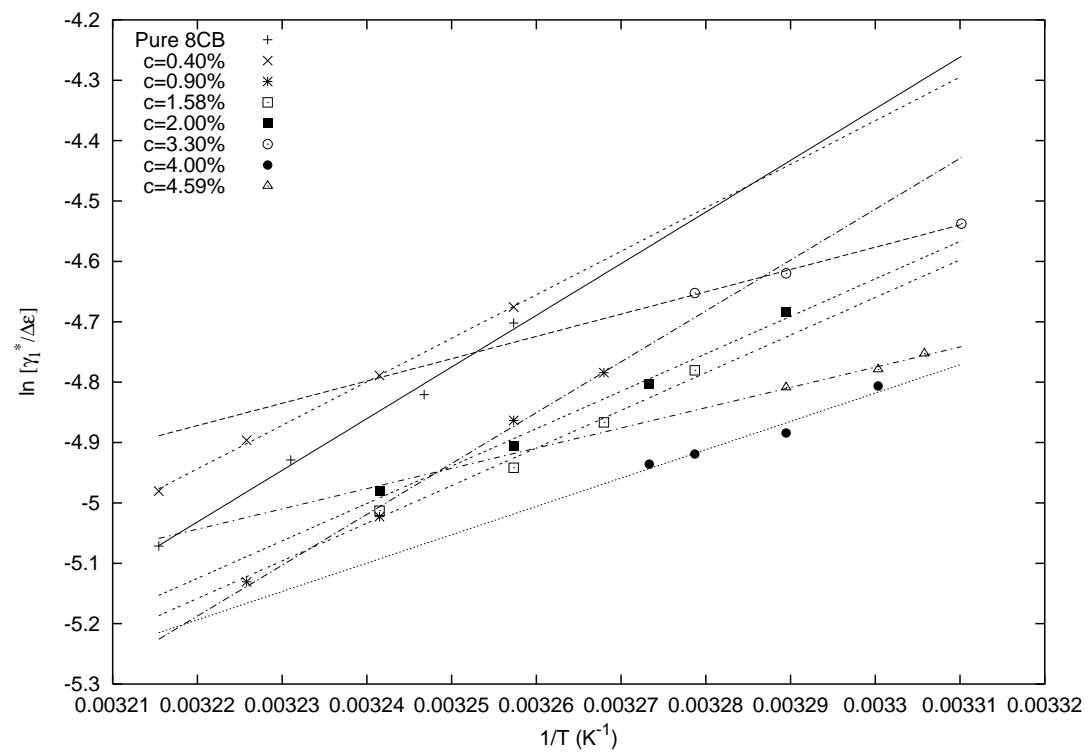

Figure 8: Variation of $\ln \left(\gamma_{1}^{*} / \Delta \epsilon\right)$ vs. $1 / T$ for different concentrations of biphenyl in $8 \mathrm{CB}$ 